ARTICLE

Received 12 Aug 2014 | Accepted 13 Nov 2014 | Published 6 Jan 2015

DOI: $10.1038 /$ ncomms 6860

OPEN

\title{
Fast photodynamics of azobenzene probed by scanning excited-state potential energy surfaces using slow spectroscopy
}

\author{
Eric M.M. Tan ${ }^{1}$, Saeed Amirjalayer ${ }^{1, \dagger}$, Szymon Smolarek ${ }^{1, \dagger}$, Alexander Vdovin ${ }^{1, \dagger}$, Francesco Zerbetto ${ }^{2}$ \\ \& Wybren Jan Buma ${ }^{1}$
}

Azobenzene, a versatile and polymorphic molecule, has been extensively and successfully used for photoswitching applications. The debate over its photoisomerization mechanism leveraged on the computational scrutiny with ever-increasing levels of theory. However, the most resolved absorption spectrum for the transition to $S_{1}\left(n \pi^{\star}\right)$ has not followed the computational advances and is more than half a century old. Here, using jet-cooled molecular beam and multiphoton ionization techniques we report the first high-resolution spectra of $S_{1}\left(n \pi^{\star}\right)$ and $S_{2}\left(\pi \pi^{\star}\right)$. The photophysical characterization reveals directly the structural changes upon excitation and the timescales of dynamical processes. For $S_{1}\left(n \pi^{\star}\right)$, we find that changes in the hybridization of the nitrogen atoms are the driving force that triggers isomerization. In combination with quantum chemical calculations we conclude that photoisomerization occurs along an inversion-assisted torsional pathway with a barrier of $\sim 2 \mathrm{kcal} \mathrm{mol}^{-1}$. This methodology can be extended to photoresponsive molecular systems so far deemed non-accessible to high-resolution spectroscopy.

\footnotetext{
${ }^{1}$ Van 't Hoff Institute for Molecular Sciences, University of Amsterdam, Science Park 904, 1098 XH Amsterdam, The Netherlands. ${ }^{2}$ Dipartimento di Chimica 'G. Ciamician', Università di Bologna, via F. Selmi 2, I-40126 Bologna, Italy. † Present addresses: Physical Institute and Center for Nanotechnology (CeNTech), Westfälische Wilhelms-Universität Münster, 48149 Münster, Germany (S.A.); ASML, De Run 6501, 5504 DR Veldhoven, The Netherlands (S.S.); Philips Research, High Tech Campus 34, 5656 AE Eindhoven, The Netherlands (A.V.). Correspondence and requests for materials should be addressed to W.J.B. (email: w.j.buma@uva.nl).
} 
ight offers unique opportunities to address molecular systems non-invasively, in a highly selective manner, and with high space and time resolution. Compounds whose shape, biological and chemical activity, dielectric constant and refractive index can be switched reversibly under the control of light thus have found extensive applications in areas that range from molecular probes to molecular nanotechnology and photopharmacology ${ }^{1-4}$. Azobenzene and its derivatives are one of the primary examples of such compounds. Their favourable properties such as photostability and functional activity under a wide range of experimental conditions have in many aspects paved the way for the further development and application of photochromic systems.

At the basis of the functional behaviour of azobenzene is the reversible trans $(\mathrm{E}) \rightarrow$ cis $(\mathrm{Z})$ isomerization of the $\mathrm{N}=\mathrm{N}$ bond upon photoexcitation. The absorption spectrum of azobenzene has therefore long attracted attention. Its low-energy part displays two bands, a weak band in the visible region $(\sim 430 \mathrm{~nm}$ in $n$-hexane) associated with excitation of the formally dipoleforbidden ${ }^{1} \mathrm{~B}_{2 \mathrm{~g}}\left(\mathrm{n} \pi^{*}\right)$ state and a strong band in the ultraviolet region ( $\sim 320 \mathrm{~nm}$ in $n$-hexane) associated with absorption of the dipole-allowed ${ }^{1} \mathrm{~B}_{1 \mathrm{u}}\left(\pi \pi^{*}\right)$ state ${ }^{5}$. One of the intriguing aspects of azobenzene photoisomerization is the dependence of the quantum yield on the excitation wavelength ${ }^{6,7}$. In principle, isomerization in azobenzene can take place along two different pathways, one that involves torsional motion around the $\mathrm{N}=\mathrm{N}$ double bond, and another in which in-plane inversion about one of the nitrogen centres takes place (Fig. 1). To accommodate the observed violation of Kasha's rule, it was therefore originally proposed that isomerization proceeds along two different pathways in the $n \pi^{*}$ and $\pi \pi^{*}$ states $^{5,8,9}$. Over the years, extensive experimental ${ }^{10-19}$ and theoretical ${ }^{20-29}$ efforts have been made to elucidate the contribution of these pathways to the photoisomerization dynamics. As yet, however, there is still no consensus on the pathway, and also the interpretation of many other aspects of the excited-state dynamics are still a matter of extensive debate.

At the basis of a fundamental understanding of the photodynamics of azobenzene is a detailed knowledge on the potential energy surfaces of the relevant excited states, preferably under isolated molecule conditions. Such studies provide detailed insight on the forces that act on the molecular structure after excitation from the ground state, map out which normal coordinates are involved in the structural dynamics, reveal whether energy barriers are involved and provide a direct

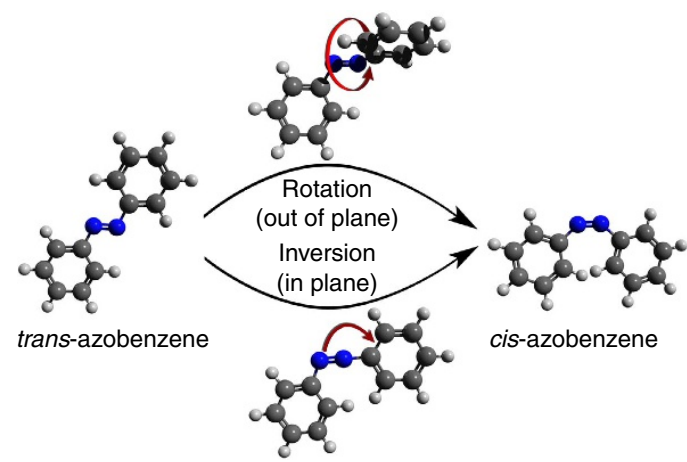

Figure 1 | Isomerization pathways of trans-azobenzene. Traditionally, isomerization of trans-azobenzene has been envisaged to occur via either a rotation or inversion mechanism. In the first mechanism torsion around the $\mathrm{N}=\mathrm{N}$ bond occurs; in the second mechanism the phenyl rings move in the plane of the molecule towards each other. benchmark for theoretical calculations ${ }^{30-33}$. Moreover, they must necessarily be the starting point for a further appreciation of the experimentally observed dependence of the photodynamics on the environment and substituents. As yet, however, azobenzene has predominantly been studied in the time domain with ultrafast laser techniques, at room temperature, and under non-isolated conditions. Under such conditions, temporal resolution is achieved at the expense of spectral resolution. As a result, the vibrational level structure of the excited state-which provides a direct fingerprint of the potential energy surface along the various normal coordinates-is not accessible.

Here, we show that advanced high-resolution two- and threecolour multiphoton ionization laser spectroscopic techniques using nanosecond lasers and molecular beams can be successfully applied to scan the potential energy surfaces of the $S_{1}\left(n \pi^{\star}\right)$ and $\mathrm{S}_{2}\left(\pi \pi^{\star}\right)$ states of trans-azobenzene and measure the vibrational structure. Such an approach is well established, but its application to azobenzene appears to contradict a priori the conditions under which such studies are normally performed. Excitation of the $\mathrm{S}_{1}\left(\mathrm{n} \pi^{\star}\right)$ is formally dipole-forbidden, and only becomes allowed when the state is vibronically coupled to another dipole-allowed excited state. In combination with an excited-state lifetime that has been reported to be in the low-picosecond regime ${ }^{3}$, resonance enhancement of the ionization yield is thus expected to be very hard to detect. Excitation of the $S_{2}\left(\pi \pi^{*}\right)$ state is fully allowed, but for this state the reported lifetime of $\sim 170 \mathrm{fs}$ in the gas phase ${ }^{9}$ severely impedes observation of resonance enhancement, and, if observed at all, lifetime broadening of resonances would be taken to preclude the detection of vibrationally resolved spectra. Despite these unfavourable conditions, we find that high-resolution spectra of both states can be obtained, and that these spectra provide a direct view on the photophysics and photochemistry of trans-azobenzene.

\section{Results}

Spectroscopy and dynamics of the $S_{1}\left(n \pi^{*}\right)$ state. Figure $2 \mathrm{a}$ shows the $S_{1}\left(n \pi^{*}\right) \leftarrow S_{0}$ excitation spectrum of trans-azobenzene in the $18,250-20,750 \mathrm{~cm}^{-1}$ region, as detected by $\left(1+1^{\prime}\right)$ resonance enhanced two-photon ionization (RE2PI) spectroscopy. Compared with the best-resolved absorption spectrum reported so far, which was obtained in mixed crystals ${ }^{34}$, the resolution of the present spectrum is several orders of magnitude better. It thereby allows for the first time a detailed analysis of the changes in molecular structure upon excitation and their implications on the photochemical activity of the molecule. Experiments and quantum chemical calculations indicate that the molecule retains $\mathrm{C}_{2 \mathrm{~h}}$ symmetry in both the ground and $\mathrm{S}_{1}\left(\mathrm{n} \pi^{\star}\right)$ state. This implies that the $S_{1}\left(n \pi^{*}\right)\left(1^{1} B_{g}\right) \leftarrow S_{0}\left(1^{1} A_{g}\right)$ transition is formally dipoleforbidden. The spectrum observed in Fig. 2a must therefore be built upon false origins involving vibrational modes of either $\mathrm{a}_{\mathrm{u}}$ or $\mathrm{b}_{\mathrm{u}}$ symmetry ${ }^{34}$ that couple the ${ }^{1} \mathrm{~B}_{\mathrm{g}}$ state to higher-lying excited singlet states of $B_{u}$ or $A_{u}$ symmetry. The first electronically excited states of $B_{u}$ and $A_{u}$ symmetry are $S_{2}$ and $S_{5}$, respectively, with calculated oscillator strengths of 0.616 and 0.008 (ref. 24). On the basis of this large difference in oscillator strengths, it is reasonable to assume that the false origins in the $S_{1}\left(n \pi^{\star}\right) \leftarrow S_{0}$ excitation spectrum involve $\mathrm{a}_{\mathrm{u}}$ vibrations, as predicted previously ${ }^{35}$ and confirmed by our experimental data (vide infra).

Analysis of the excitation spectrum leads to the conclusion that it consists of several false origins upon which vibrational progressions involving totally symmetric $\mathrm{a}_{\mathrm{g}}$ modes are built (see Supplementary Table 1), in particular $v_{23}\left(188 \mathrm{~cm}^{-1}\right), v_{22}$ $\left(294 \mathrm{~cm}^{-1}\right), v_{20}\left(653 \mathrm{~cm}^{-1}\right)$ and $v_{19}\left(853 \mathrm{~cm}^{-1}\right)$. Importantly, we observe that $v_{23}$, which contains a significant amount of the 


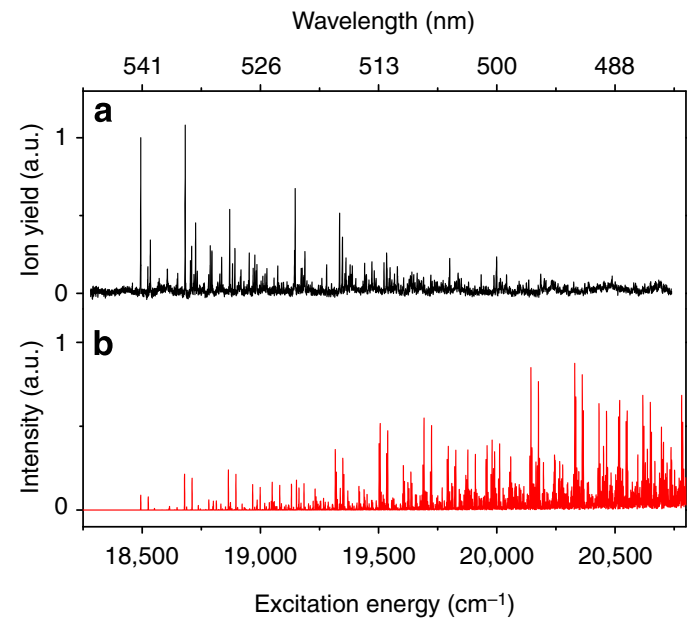

Figure 2 | Excitation spectrum of $S_{1}\left(n \pi^{\star}\right) \leftarrow S_{0}$ transition of

trans-azobenzene. (a) Experimental jet-cooled excitation spectrum obtained using two-colour resonance enhanced two-photon ionization spectroscopy. The horizontal axis labels the frequency of the laser used for excitation of the molecule to the $S_{1}\left(n \pi^{\star}\right)$ state, which is subsequently ionized by absorption of a 193-nm photon. (b) Excitation spectrum predicted within the Herzberg-Teller approximation of the electronic transition dipole moment using quantum chemical calculations of the equilibrium geometries, harmonic force fields of the ground and $S_{1}\left(n \pi^{\star}\right)$ excited state, as well as electronic transition dipole derivatives. The spectrum has been generated by convolution of calculated stick spectra with $0.4-\mathrm{cm}^{-1}$ bands, as found experimentally for the initial part of the excitation spectrum (see text).

NNC bend character, is the most active totally symmetric mode. This mode is directly associated with in-plane inversion of the two nitrogen atoms. Its motion thus relates directly to one of the suggested photoisomerization mechanisms, albeit that the actual photoisomerization process would involve a non-totally symmetric inversion mode. Nevertheless, the dominant activity of $v_{23}$ is significant, as it indicates that the hybridization and the related angles of the nitrogen atoms change considerably upon excitation. The importance of geometry changes along the inversion coordinate is also reflected in the activity of $v_{20}$, the secondmost active mode. This mode involves predominantly CCC bending motions of the phenyl rings, but for a large part also NNC-bending motions.

Figure $2 \mathrm{~b}$ displays the $\mathrm{S}_{1}\left(\mathrm{n} \pi^{*}\right) \leftarrow \mathrm{S}_{0}$ excitation spectrum predicted within the Herzberg-Teller approximation ${ }^{36}$. If we consider for the moment only the vibrational progressions involving totally symmetric $a_{g}$ modes, qualitatively good agreement is observed between experimental and predicted intensities (see also Supplementary Table 4), although quantitatively the differences are larger than what one would have expected on the basis of studies of similar complexity ${ }^{37,38}$. In general, such differences indicate that the geometry changes upon excitation are not predicted accurately enough. To get an idea of the geometry changes that would lead to consistency with the experimentally observed excitation spectrum, we have employed the observed intensities of $v_{i}\left(a_{g}\right)_{0}^{1}$ transitions to 'reconstruct' the equilibrium geometry of the $S_{1}\left(n \pi^{*}\right)$ state ${ }^{39,40}$. In our experiments, we primarily observe Franck-Condon activity of bending vibrations and not of $\mathrm{CC}$ and $\mathrm{CN}$ stretch vibrations, which, as will be argued below, is attributed to the opening up of an additional non-radiative decay channel at these vibrational energies. As a result, the reconstructed geometry primarily reflects changes in the bond angles and not in bond lengths. Table 1 reports the most relevant geometrical parameters of ground and excited states as originally predicted by our calculations-which are in excellent agreement with previous calculations ${ }^{21,26}$-and as reconstructed from the excitation spectrum. This table indicates that the change in the NNC bond angle is considerably smaller than what is predicted by the calculations.

Above we have argued that the false origins are in principle associated with $a_{u}$ or $b_{u}$ vibrations. Consideration of the frequency intervals between the false origins and comparison with calculated vibrational frequencies rapidly leads to two conclusions. First, we find that these frequency intervals are in excellent agreement with what one would expect for the manifold of $a_{u}$ vibrations, but cannot be reconciled with the frequencies of $b_{u}$ vibrations (see Supplementary Table 3). Further support for an assignment to $a_{u}$ vibrations comes from simulations of the rotational contours of the bands (vide infra). These contours can be reproduced very well under the assumption of a $B_{u}$ $\left(B_{g} \otimes a_{u}\right) \leftarrow A_{g}$ vibronic transition, but completely fail for an $A_{u}$ $\left(B_{g} \otimes b_{u}\right) \leftarrow A_{g}$ vibronic transition. We conclude that the $\mathrm{S}_{1}\left(\mathrm{n} \pi^{\star}\right) \leftarrow \mathrm{S}_{0}$ transition obtains its intensity by vibronic coupling with the $1^{1} \mathrm{~B}_{\mathrm{u}}$ state, in agreement with a priori expectations. Second, it has to be concluded that the strong band at $18,493.8 \mathrm{~cm}^{-1}$ is the lowest-energy false origin and is associated with $v_{44}$, the lowest-frequency $a_{u}$ vibration. The true $S_{1}\left(n \pi^{*}\right) \leftarrow S_{0}$ 0-0 origin transition is not observed, but we can obtain a reasonably accurate estimate of its value by two different approaches, which both lead to the conclusion that it occurs at $18,471.7 \mathrm{~cm}^{-1}$ (see Supplementary Notes 1 and 2).

The $S_{1}\left(n \pi^{*}\right) \leftarrow S_{0}$ excitation spectrum does not show only the $44_{0}^{1}$ false origin but also false origins associated with the $44_{0}^{3}$ and $44_{0}^{5}$ transitions. These transitions are forbidden within the harmonic oscillator approximation, and can only obtain intensity by anharmonicity or Duschinsky mixing, that is, normal-mode rotation upon excitation. As the energy differences between the bands indicate that the anharmonicity is rather small, one has to conclude that Duschinsky mixing is the principal reason for the appearance of the overtone false origins. Quantum chemical calculations confirm this conclusion: they show that $v_{44}$ and $v_{43}$ become extensively mixed upon excitation.

Apart from $v_{44}$, several other $a_{u}$ modes serve as false origins as well, most notably $v_{43}, v_{42}, v_{41}, v_{40}$ and $v_{37}$ whose transitions are displaced from the $S_{1}(n \pi *) \leftarrow S_{0} 44_{0}^{1}$ false origin by $29.9,210.3$, $388.3,459.0$ and $786.4 \mathrm{~cm}^{-1}$, respectively. Comparison of the relative intensities of the false origins (see Supplementary Table 5) shows that $v_{44}$ is by far the most effective in inducing intensity in the $S_{1}\left(n \pi^{*}\right) \leftarrow S_{0}$ transition. It is well known that vibronic coupling leads to changes in the frequency of the coupling mode ${ }^{41,42}$. Compared with the uncoupled case, the frequency of the coupling mode is reduced in the lower state and increased in the upper state. Theory thus makes one expect that the relative frequency difference in the two states is a good measure for the vibronic coupling strength. Indeed, we find that the frequency of $v_{44}$ in $S_{1}$ and $S_{2}$ differs by more than a factor of two, while the other modes show much more moderate differences (see Supplementary Table 3). The conclusion that the $S_{1}\left(n \pi^{*}\right) \leftarrow S_{0}$ transition is to a dominant extent enabled by $v_{44}$ is an important one. This mode is essentially a pure CNNC torsional mode, the mode along which isomerization has been proposed to occur. The overall picture that thus emerges from the present isolated molecule spectrum is one in which the $S_{1}\left(n \pi^{*}\right)$ state can only be accessed if simultaneously an $a_{u}$ vibrational mode is excited, and that the most effective way to do so is to impart energy into the mode that involves torsion around the $\mathrm{N}=\mathrm{N}$ double bond. It should be kept in mind that the present experiments have been performed under conditions in which vibrational levels in the ground state are not populated. At room temperature, however, low-frequency vibrations will be excited as well. Under such 
Table 1 | Relevant geometrical parameters of trans-azobenzene in the $S_{0}, S_{1}\left(n \pi^{\star}\right)$ and $S_{2}\left(\pi \pi^{\star}\right)$ states as determined from the experiment and predicted by quantum chemical calculations.

\begin{tabular}{|c|c|c|c|c|c|}
\hline & $S_{0}$ experiment $^{\star}$ & $S_{1}$ theory ${ }^{\dagger}$ & $\mathbf{S}_{1}$ reconstructed ${ }^{\dagger, \ddagger}$ & $S_{2}$ theory ${ }^{\dagger}$ & $S_{2}$ reconstructed ${ }^{\S}$ \\
\hline$d(N=N)(\AA)$ & 1.260 & 1.251 & 1.262 & 1.351 & 1.324 \\
\hline$\angle N=N-C\left(^{\circ}\right)$ & 113.7 & 130.5 & 124.3 & 111.5 & 112.2 \\
\hline
\end{tabular}

${ }^{\star}$ Structural parameters taken from gas-phase electron diffraction 49

†Present work.

$\ddagger$ Determined from experimental intensities of $v_{i}\left(a_{0}\right)_{0}^{1}$ transitions for modes $i=23-19$, and the intensities predicted by the calculations for modes $i=18-6$ (see Supplementary Table 4).

\$Determined from experimental intensities of $v_{i}\left(a_{q}\right)_{0}^{1}$ transitions for modes that have been observed and identified in the $S_{2}\left(\pi \pi^{*}\right) \leftarrow S_{0}$ spectrum. Modes that have not been observed have been given zero intensity (see Supplementary Table 4).

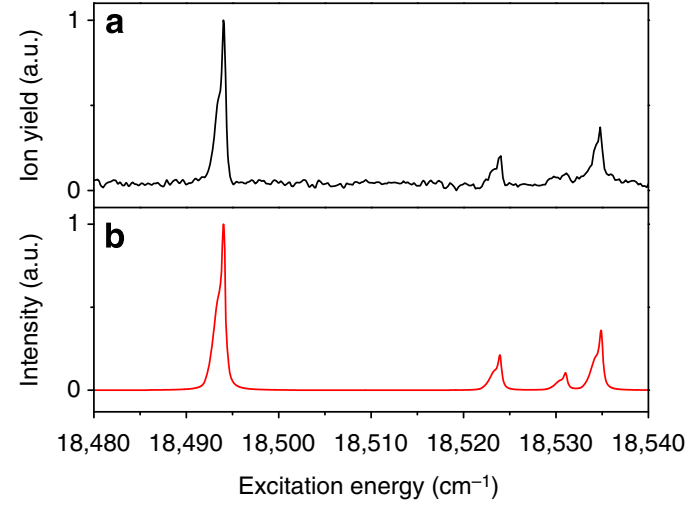

Figure 3 | Rotational contour of bands in the $S_{1}\left(n \pi^{\star}\right) \leftarrow S_{0}$ excitation spectrum near the forbidden 0-0 transition. (a) Experimentally observed contours. (b) Simulated contours assuming a vibronic $B_{u} \leftarrow A_{g}$ transition, a rotational temperature of $9 \mathrm{~K}$ and a homogeneous linewidth for individual rotational transitions of $0.4 \mathrm{~cm}^{-1}$.

conditions, transitions can also occur in which the $\mathrm{a}_{\mathrm{u}}$ vibrational quantum number is decreased ${ }^{35}$.

Figure 3 shows fits of the rotational contour of bands in the initial part of the excitation spectrum that start from the vibrationless ground state and use rotational constants and electronic transition moments as calculated. Excellent agreement between the experiment and simulation is observed, leading to the conclusion that under our experimental conditions the rotational population distribution can be described by a rotational temperature of $9 \mathrm{~K}$. More importantly, these simulations show that the homogeneous linewidth of the individual rotational transitions is $0.4 \mathrm{~cm}^{-1}$ corresponding to a lifetime of the lower rovibronic levels of $S_{1}$ of about 13 ps (see Supplementary Note 3 for a further discussion of the fits). This lifetime is almost an order of magnitude longer than the lifetime reported so far in solution-phase experiments on the $S_{1}\left(n \pi^{\star}\right)$ state $11,12,14,16$. The large difference between gas- and solution-phase experiments is remarkable, but, as argued below, is completely in line with the different experimental conditions under which the two types of experiments have been performed.

Figure 4 shows the dependence of the linewidths on the excitation energy, as determined from fits of the rotational contours. Although there is considerable scatter among the linewidths, it is clear that for low excitation energies the bands have a similar linewidth. However, for excitation energies above $\sim 19,250-19,500 \mathrm{~cm}^{-1}$ the linewidths become larger, increasing to values of $4 \mathrm{~cm}^{-1}$ and larger around $20,500 \mathrm{~cm}^{-1}$. These observations strongly suggest that the decay dynamics of the $\mathrm{S}_{1}\left(\mathrm{n} \pi^{\star}\right)$ state involve a non-radiative decay channel that opens up at about $750-1,000 \mathrm{~cm}^{-1}$ above the vibrationless level of the

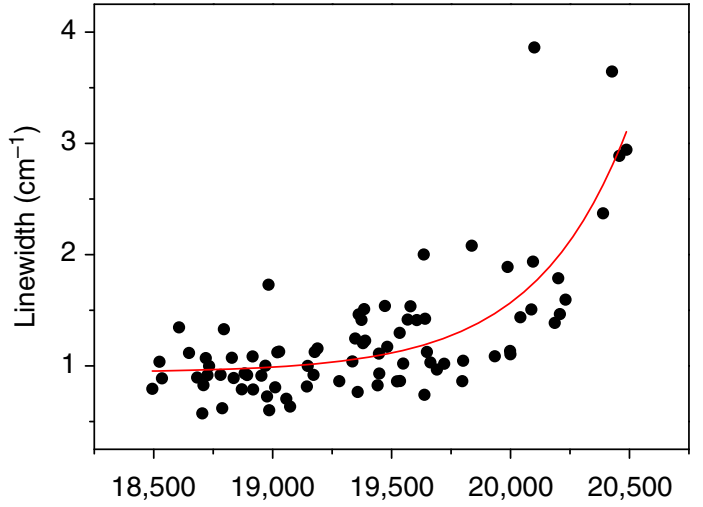

Peak center $\left(\mathrm{cm}^{-1}\right)$

Figure 4 | Influence of excitation energy on linewidths. Dependence of linewidths of bands in the $S_{1}\left(n \pi^{\star}\right) \leftarrow S_{0}$ excitation spectrum on excitation energy, the drawn line serving as a guide to the eye. For low excitation energies, bands have rather similar linewidths, but these start to increase markedly above $\sim 19,250-19,500 \mathrm{~cm}^{-1}$. This increase gives evidence for an additional non-radiative decay channel that opens up for excess energies of $\sim 2 \mathrm{kcal} \mathrm{mol}^{-1}$

$S_{1}\left(n \pi^{*}\right)$ state. The same conclusion is reached when the experimentally observed vibronic intensities (Fig. 2a) are compared with theoretically predicted intensities (Fig. 2b). Up to $\sim 19,250-19,500 \mathrm{~cm}^{-1}$ experiment and theory match well, but for higher excitation energies the experimental spectrum shows a progressive decrease of vibronic activity while theory predicts in contrast an increase in activity. Franck-Condon considerations for the ionization step of our experiments exclude any nonradiative decay mechanism that leaves azobenzene in an electronically excited state. Our experiments therefore show that the marked loss in intensity in the experimental spectrum is due to a very fast decay to the ground state.

The presence of a decay channel that only comes into play above a certain threshold energy explains the apparent mismatch between the lifetime measured in the present experiments and in solution. Time-resolved transient absorption and fluorescence experiments in solution differ in two aspects from the present experiments. First and most importantly, in solution molecules are excited at or near the absorption maximum, leading to excitation energies that are at least some $2,000 \mathrm{~cm}^{-1}$ above the 0-0 transition determined here. Second, the present experiments have been performed at an effective rotational temperature of $9 \mathrm{~K}$-the vibrational temperature admittedly being somewhat higher-while solution-phase experiments have been performed at room temperature. Both conditions lead to a situation in which the solution-phase experiments' vibronic levels are excited in a 
region well above the barrier for accessing the non-radiative decay channel discussed above. On a timescale of a few ps the effects of vibrational cooling are still limited ${ }^{43}$, and one would therefore expect that in the solution-phase experiments decay times are observed that are near the values estimated here for excitation of high-lying vibronic levels. This expectation is borne out by our experiments: the reported range of decay times of $0.6-2.6 \mathrm{ps}$ corresponds to linewidths of $2-9 \mathrm{~cm}^{-1}$.

The photoisomerization mechanism of azobenzene after excitation of the $S_{1}\left(n \pi^{*}\right)$ state is still a subject of extensive debate. In the present study, we do not have direct access to the reactive part of the potential energy surface, but we do notice that our observations agree remarkably well with the relevant features of the potential energy surface as emerging from high-level $a b$ initio calculations ${ }^{24,26}$. This pertains in particular to the presence of a $\sim 750-1,000-\mathrm{cm}^{-1}\left(2.1-2.9 \mathrm{kcal} \mathrm{mol}^{-1}\right)$ barrier for accessing an additional non-radiative decay channel. Calculations find on the potential energy surface of the $S_{1}\left(n \pi^{*}\right)$ state a transition state with an activation energy of about $2 \mathrm{kcal} \mathrm{mol}^{-1}$. Conical intersections of $S_{1}$ and $S_{0}$ along the torsional pathway are found at more or less the same energy, while conical intersections along the inversion pathway occur at much higher energies ${ }^{22,24}$. We thus conclude that the experimentally observed barrier is in excellent agreement with the predicted activation energy, while the experimental conclusion that above the barrier the excited state decays ultrafast to the electronic ground state is entirely consistent with an $S_{0} / S_{1}$ conical intersection at similar energies as the activation energy. Taking into account that both experiment and theory agree that excitation is accompanied by a significant change of the NNC bond angle, it would appear that for the isolated molecule photoisomerization predominantly takes place via an inversion-assisted torsional pathway ${ }^{3}$ in which large changes in the CNNC dihedral angle occur simultaneously with smaller, but significant changes in the NNC angles. Importantly, changes along the CNNC torsional coordinate are intrinsically already promoted by the fact that excitation of the $S_{1}\left(n \pi^{\star}\right)$ state occurs predominantly by simultaneously exciting the $\mathrm{N}=\mathrm{N}$ double-bond torsional vibration.

Spectroscopy and dynamics of the $S_{2}\left(\pi \pi^{\star}\right)$ state. The excitation spectrum of jet-cooled trans-azobenzene to the second excited state is shown in Fig. 5a. We find that the $S_{2}\left(\pi \pi^{*}\right) \leftarrow S_{0} 0_{0}^{0}$ transition is located at $30,096.6 \pm 0.2 \mathrm{~cm}^{-1}$. Qualitatively, the spectrum is similar to previously reported spectra of azobenzene in dibenzyl crystals ${ }^{44}$ and in the vapour phase ${ }^{45,46}$, but the resolution is significantly higher. This resolution in fact enables us to conclude that the widths of the bands are lifetime limited. Fits of the origin band with a Lorentzian find a width of $32.0 \pm 1.0 \mathrm{~cm}^{-1}$ corresponding to a lifetime of $166 \pm 5 \mathrm{fs}$, which is in excellent agreement with the lifetime reported in femtosecond time-resolved photoelectron spectroscopic studies $(170 \mathrm{fs})^{9}$, and time-resolved fluorescence studies $(110 \mathrm{fs})^{47}$.

Figure 5a shows that the most active mode in the $S_{2}\left(\pi \pi^{*}\right) \leftarrow S_{0}$ spectrum is $v_{23}\left(223 \mathrm{~cm}^{-1}\right)$, similar to what is observed for excitation of the $S_{1}\left(n \pi^{\star}\right)$ state. In agreement with the previous low-resolution studies ${ }^{44}$, we also see a prominent role of $v_{12}$ $\left(1,315 \mathrm{~cm}^{-1}\right)$. However, at the same time the present resolution enables us to observe the activity of many other modes that so far have not been reported, and thereby to determine in greater detail the structural changes occurring upon excitation to this state. Detailed assignment of the bands in the excitation spectrum (see Supplementary Table 2) shows that they nearly all involve fundamentals of totally symmetric vibrations and their combinations. The only exception is the low-intensity band at $103 \mathrm{~cm}^{-1}$ from the origin, which also appears in combination

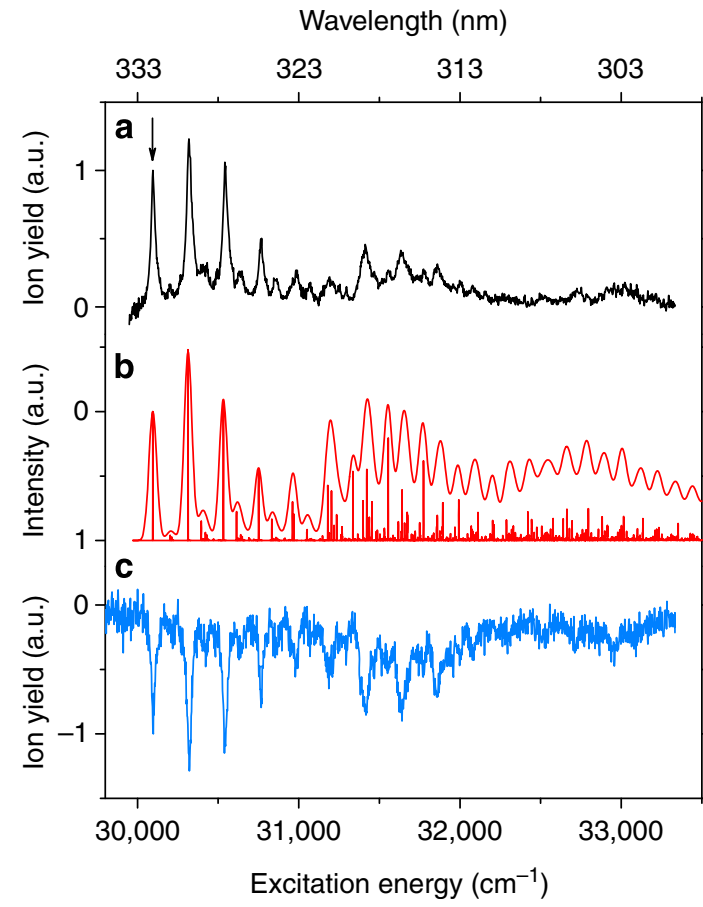

Figure 5 | Excitation spectrum of $S_{2}\left(\pi \pi^{\star}\right) \leftarrow S_{0}$ transition of trans-azobenzene. (a) Experimental jet-cooled excitation spectrum obtained using two-colour resonance enhanced two-photon ionization spectroscopy. The horizontal axis labels the frequency of the laser used for excitation of the molecule to the $S_{2}\left(\pi \pi^{\star}\right)$ state, which is subsequently ionized by absorption of a 193-nm photon. (b) Excitation spectrum predicted within the Franck-Condon approximation using quantum chemical calculations of the equilibrium geometries and harmonic force fields of the ground and $S_{2}\left(\pi \pi^{\star}\right)$ excited states. The spectrum has been generated by convolution of calculated stick spectra with $32-\mathrm{cm}^{-1}$ bands, as found experimentally for the initial part of the excitation spectrum (see text). (c) Ultraviolet-ultraviolet depletion spectrum of the $S_{2}\left(\pi \pi^{\star}\right)$ state using the transition at $30,096.6 \mathrm{~cm}^{-1}$ indicated by the arrow as a probe.

with other bands. This band must be attributed to the $44_{0}^{2}$ transition. The activity of this mode indicates that it changes considerably upon excitation, as was also concluded for the $\mathrm{S}_{1}\left(\mathrm{n} \pi^{\star}\right)$ state.

Comparison of the experimental spectrum with the theoretically predicted spectrum (Fig. 5b) shows very good agreement, and indicates that the broadening of the experimental spectrum at higher energy is due to spectral congestion. In contrast to simulations of the $S_{1}\left(n \pi^{\star}\right) \leftarrow S_{0}$ spectrum, the predicted intensities now follow quite closely the experimentally observed intensities. Accordingly, we find that reconstruction of the equilibrium geometry of the $S_{2}\left(\pi \pi^{*}\right)$ state from the experimentally observed intensities leads to minor changes in bond lengths and bond angles (Table 1).

The relaxation pathways and dynamics of the $S_{2}\left(\pi \pi^{\star}\right)$ state have been the subject of a large amount of experimental and theoretical studies. In several of these studies, it has been suggested that other electronically excited states are involved in the decay of the $S_{2}\left(\pi \pi^{*}\right)$ state $9,24,26$. Generally, different electronic states have different ionization cross-sections. One might thus see signatures of an excitation-energy-dependent involvement of other electronically excited states by comparing the $\left(1+1^{\prime}\right)$ RE2PI excitation spectrum-for which intensities depend on both excitation and ionization cross-section-with the absorption spectrum of the $S_{2}\left(\pi \pi^{\star}\right)$ state obtained by ultraviolet-ultraviolet depletion spectroscopy for which intensities only depend on the 
excitation cross-section. However, comparison of the ultravioletultraviolet depletion spectrum shown in Fig. 5c with the RE2PI spectrum depicted in Fig. 5a does not give any indication of significant differences between the two spectra. This similarity also precludes significant contributions from decay channels leading to states that cannot be ionized, as would occur upon internal conversion to the ground state. We therefore conclude that our observations are consistent with an efficient ultrafast $S_{1} \leftarrow S_{2}$ internal conversion, but cannot make definite statements on further details of this pathway.

\section{Discussion}

High-resolution nanosecond laser spectroscopy has enabled us to scan the potential energy surfaces of electronically excited states of trans-azobenzene. These studies have unveiled key aspects of how the structure of the molecule is changed upon photoexcitation, and how photoisomerization takes place. From these studies a picture has been established in which (a) excitation of the $S_{1}\left(n \pi^{\star}\right)$ state is primarily accompanied by in-plane changes in the hybridization angles around the nitrogen atoms, (b) excitation of this dipole-forbidden state occurs most effectively if it involves the CNNC torsional mode, the mode that is directly involved in the torsional isomerization pathway and (c) photoisomerization in the $S_{1}\left(n \pi^{*}\right)$ state occurs via an inversion-assisted torsional pathway with a barrier of $\sim 2 \mathrm{kcal} \mathrm{mol}^{-1}$ separating the local minimum of the $S_{1}\left(n \pi^{\star}\right)$ state from a conical intersection with the ground state at similar energies as the barrier. Similarly, we have determined the structural changes occurring upon excitation to the strongly absorbing $S_{2}\left(\pi \pi^{*}\right)$ state. Comparison of the absorption and excitation spectra of this state have led to the conclusion that, at least from these spectra, no indication can be found for the importance of dynamical processes other than internal conversion to the $S_{1}\left(n \pi^{\star}\right)$ state.

Elucidation of the details of the potential energy surfaces of the $S_{1}\left(n \pi^{*}\right)$ and $S_{2}\left(\pi \pi^{*}\right)$ states and their associated dynamics is important, since they ultimately determine the operation of azobenzene in photofunctional devices. In this respect, the present results could form the basis for a further rational design of novel molecular devices that use this important building block. At a more general level, the present studies have shown the important benefits high-resolution spectroscopy can offer for the study of (ultra)fast photodynamics. They should provide further impetus for similar studies on the vast amount of photoresponsive systems that so far have not been considered, because their lifetimes were considered to be prohibitively short.

\footnotetext{
Methods

Experimental details. In our studies, excitation spectra of cold isolated molecules were recorded by two-colour $\left(1+1^{\prime}\right)$ RE2PI spectroscopy on trans-azobenzene seeded in a supersonic expansion of neon. For this purpose, trans-azobenzene (Sigma-Aldrich) was placed in an oven that was heated up to $145^{\circ} \mathrm{C}$ and expanded into vacuum with 2 bars of $\mathrm{Ne}$ via a pulsed nozzle with a $0.5-\mathrm{mm}$ orifice (General Valve Iota One system). Following the supersonic free jet expansion, a 2-mm conical skimmer skimmed the expansion prior to entering the ionization chamber. Ions were detected using a reflectron time-of-flight mass spectrometer (R.M. Jordan Co.).

In the two-colour RE2PI experiments, a frequency-doubled dye laser (Sirah Precision Scan) pumped by a Nd:YAG laser (Spectra Physics Lab 190) operating at $30 \mathrm{~Hz}$ was used for excitation. Typical pulse energies were $2-3 \mathrm{~mJ}$, but when necessary these were attenuated to avoid saturation. Ionization occurred by means of an ArF excimer laser (Neweks PSX-501) that typically produces pulses with energies of $5 \mathrm{~mJ}$ per pulse. The two lasers are introduced into the spectrometer in a counter-propagating fashion without further focusing. The pulsed nozzle and the two laser systems were synchronized by a delay generator (Stanford Research Systems DG535). For the ultraviolet-ultraviolet depletion experiments, another laser system consisting of a frequency-doubled dye laser (Sirah Cobra-Stretch) pumped by a second Nd:YAG laser (Spectra Physics Lab 190) was employed. In these experiments, the depletion laser, which is scanned over the excitation energy of interest, was fired $100-200 \mathrm{~ns}$ prior to the lasers creating the $\left(1+1^{\prime}\right)$ RE2PI
} probe signal at a fixed excitation wavelength.
Computational details. Geometry optimization followed by calculation of the harmonic force field was performed for the $S_{0}$ ground state and the $S_{1}\left(n \pi^{*}\right)$ and $\mathrm{S}_{2}\left(\pi \pi^{*}\right)$ electronically excited states using time-dependent density functional theory. These calculations were performed at the B3LYP/6-31G(d,p) level utilizing the Gaussian09 suite of programs ${ }^{48}$. The same program was employed for simulation of the vibrationally resolved excitation spectra within the Herzberg-Teller approximation. For comparison with experimental spectra, a scaling factor of 0.961 was used for the predicted frequencies.

\section{References}

1. Browne, W. R. \& Feringa, B. L. Light switching of molecules on surfaces. Annu. Rev. Phys. Chem. 60, 407-428 (2009).

2. Fehrentz, T., Schönberger, M. \& Trauner, D. Optochemical genetics. Angew. Chem. Int. Ed. 50, 12156-12182 (2011).

3. Bandara, H. M. D. \& Burdette, S. C. Photoisomerization in different classes of azobenzene. Chem. Soc. Rev. 41, 1809-1825 (2012).

4. Pathem, B. K., Claridge, S. A., Zheng, Y. B. \& Weiss, P. S. Molecular switches and motors on surfaces. Annu. Rev. Phys. Chem. 64, 605-630 (2013).

5. Rau, H. in Photochromism: Molecules and Systems Vol. 1 (eds Dür, H. \& Boumas-Laurent, H.) 165-192 (Elsevier, 1990).

6. Bortolus, P. \& Monti, S. Cis-trans photoisomerization of azobenzene. Solvent and triplet donors effects. J. Phys. Chem. 83, 648-652 (1979).

7. Siampiringue, N., Guyot, G., Monti, S. \& Bortolus, P. The cis $\rightarrow$ trans photoisomerization of azobenzene: an experimental re-examination. J. Photochem. 37, 185-188 (1987).

8. Rau, H. \& Lueddecke, E. On the rotation-inversion controversy on photoisomerization of azobenzenes. Experimental proof of inversion. J. Am. Chem. Soc. 104, 1616-1620 (1982).

9. Schultz, T. et al. Mechanism and dynamics of azobenzene photoisomerization. J. Am. Chem. Soc. 125, 8098-8099 (2003).

10. Lednev, I. K., Ye, T.-Q., Hester, R. E. \& Moore, J. N. Femtosecond timeresolved UV - visible absorption apectroscopy of trans-azobenzene in solution. J. Phys. Chem. 100, 13338-13341 (1996).

11. Nägele, T., Hoche, R., Zinth, W. \& Wachtveitl, J. Femtosecond photoisomerization of cis-azobenzene. Chem. Phys. Lett. 272, 489-495 (1997).

12. Lednev, I. K. et al. Femtosecond time-resolved UV-visible absorption spectroscopy of trans-azobenzene: dependence on excitation wavelength. Chem. Phys. Lett. 290, 68-74 (1998).

13. Fujino, T. \& Tahara, T. Picosecond time-resolved Raman study of transazobenzene. J. Phys. Chem. A 104, 4203-4210 (2000).

14. Satzger, H. et al. Fluorescence spectra of trans- and cis-azobenzene-emission from the Franck-Condon state. Chem. Phys. Lett. 372, 216-223 (2003).

15. Satzger, H., Root, C. \& Braun, M. Excited-state dynamics of trans- and cis-azobenzene after UV excitation in the $\pi \pi^{*}$ band. J. Phys. Chem. A 108, 6265-6271 (2004).

16. Chang, C.-W., Lu, Y.-C., Wang, T.-T. \& Diau, E. W.-G. Photoisomerization dynamics of azobenzene in solution with $S_{1}$ excitation: a femtosecond fluorescence anisotropy study. J. Am. Chem. Soc. 126, 10109-10118 (2004).

17. Stuart, C. M., Frontiera, R. R. \& Mathies, R. A. Excited-state structure and dynamics of cis- and trans-azobenzene from resonance Raman intensity analysis. J. Phys. Chem. A 111, 12072-12080 (2007).

18. Chowdary, P. D. \& Umapathy, S. Ultrafast dynamics and photochemistry of $\pi-\pi^{*}$ excited trans-azobenzene-a comprehensive analysis of resonance Raman intensities. J. Raman Spectrosc. 39, 1538-1555 (2008).

19. Quick, M. et al. Photoisomerization dynamics and pathways of trans- and cis-azobenzene in solution from broadband femtosecond spectroscopies and calculations. J. Phys. Chem. B 118, 8756-8771 (2014).

20. Ishikawa, T., Noro, T. \& Shoda, T. Theoretical study on the photoisomerization of azobenzene. J. Chem. Phys. 115, 7503-7512 (2001)

21. Cembran, A., Bernardi, F., Garavelli, M., Gagliardi, L. \& Orlandi, G. On the mechanism of the cis - trans isomerization in the lowest electronic states of azobenzene: $\mathrm{S}_{0}, \mathrm{~S}_{1}$, and $\mathrm{T}_{1}$. J. Am. Chem. Soc. 126, 3234-3243 (2004).

22. Wei-Guang Diau, E. A new trans-to-cis photoisomerization mechanism of azobenzene on the $\mathrm{S}_{1}\left(\mathrm{n}, \pi^{*}\right)$ surface. J. Phys. Chem. A 108, 950-956 (2004).

23. Gagliardi, L., Orlandi, G., Bernardi, F., Cembran, A. \& Garavelli, M. A theoretical study of the lowest electronic states of azobenzene: the role of torsion coordinate in the cis-trans photoisomerization. Theor. Chem. Acc. 111, 363-372 (2004).

24. Conti, I., Garavelli, M. \& Orlandi, G. The different photoisomerization efficiency of azobenzene in the lowest $n \pi^{*}$ and $\pi \pi^{*}$ singlets: the role of a phantom state. J. Am. Chem. Soc. 130, 5216-5230 (2008).

25. Yuan, S., Dou, Y., Wu, W., Hu, Y. \& Zhao, J. Why does trans-azobenzene have a smaller isomerization yield for $\pi \pi^{\star}$ excitation than for $\mathrm{n} \pi^{\star}$ excitation? J. Phys Chem. A 112, 13326-13334 (2008).

26. Wang, L., Xu, W., Yi, C. \& Wang, X. Isomerization and electronic relaxation of azobenzene after being excited to higher electronic states. J. Mol. Graph. Model. 27, 792-796 (2009) 
27. Tiberio, G., Muccioli, L., Berardi, R. \& Zannoni, C. How does the trans-cis photoisomerization of azobenzene take place in organic solvents? Chemphyschem 11, 1018-1028 (2010).

28. Cusati, T., Granucci, G. \& Persico, M. Photodynamics and time-resolved fluorescence of azobenzene in solution: a mixed quantum-classical simulation. J. Am. Chem. Soc. 133, 5109-5123 (2011).

29. Harabuchi, Y., Ishii, M., Nakayama, A., Noro, T. \& Taketsugu, T. A multireference perturbation study of the NN stretching frequency of trans-azobenzene in $\mathrm{n} \pi^{*}$ excitation and an implication for the photoisomerization mechanism. J. Chem. Phys. 138, 064305 (2013).

30. Ferrante, C., Kensy, U. \& Dick, B. Does diphenylacetylene (tolan) fluoresce from its second excited singlet state? Semiempirical MO calculations and fluorescence quantum yield measurements. J. Phys. Chem. 97, 13457-13463 (1993).

31. Heikal, A. A., Baskin, J. S., Bañares, L. \& Zewail, A. H. Structural effects on the isomerization dynamics of trans-stilbenes: IVR, microcanonical reaction rates, and the nature of the transition state. J. Phys. Chem. A 101, 572-590 (1997).

32. Müller, C., Klöppel-Riech, M., Schröder, F., Schroeder, J. \& Troe, J. Fluorescence and REMPI spectroscopy of jet-cooled isolated 2-phenylindene in the $\mathrm{S}_{1}$ state. J. Phys. Chem. A 110, 5017-5031 (2006).

33. Tan, E. M. M., Amirjalayer, S., Bakker, B. H. \& Buma, W. J. Excited state dynamics of photoactive yellow protein chromophores elucidated by highresolution spectroscopy and ab initio calculations. Faraday Discuss. 163, 321-340 (2013).

34. Hochstrasser, R. M. \& Lower, S. K. Polarization of the spectra of crystalline azobenzene and mixed crystals of azobenzene in stilbene at $77^{\circ}$ and $4.2^{\circ} \mathrm{K}$ in the region of the lowest $\mathrm{n} \rightarrow \pi^{\star}$ transition. J. Chem. Phys. 36, 3505-3506 (1962).

35. Cusati, T., Granucci, G., Persico, M. \& Spighi, G. Oscillator strength and polarization of the forbidden $\mathrm{n} \rightarrow \pi^{*}$ band of trans-azobenzene: a computational study. J. Chem. Phys. 128, 194312 (2008).

36. Herzberg, G. \& Teller, E. Schwingungsstruktur der Electronenübergange bei mehratomigen Molekülen. Z. Phys. Chem. B 21, 410-446 (1933).

37. Buma, W. J. \& Zerbetto, F. Vibronic activity in trans,trans-1,3,5,7 octatetraene: the $\mathrm{S}_{0} \rightarrow \mathrm{S}_{1}$ spectrum. J. Chem. Phys. 103, 10492-10501 (1995).

38. Buma, W. J. \& Zerbetto, F. Modeling the spectroscopy of the lowest excited singlet state of cis,trans-1,3,5,7-octatetraene: the role of symmetry breaking and vibronic interactions. J. Phys. Chem. A 103, 2220-2226 (1998).

39. Doktorov, E. V., Malkin, I. A. \& Man'ko, V. I. Dynamical symmetry of vibronic transitions in polyatomic molecules and the Franck-Condon principle. J. Mol. Spectrosc. 64, 302-326 (1977).

40. Zwier, J. M., Wiering, P. G., Brouwer, A. M., Bebelaar, D. \& Buma, W. J. The lowest excited singlet states of 1-azaadamantane and 1-azabicyclo[2.2.2]octane: fluorescence excitation spectroscopy and density functional calculations. J. Am. Chem. Soc. 119, 11523-11533 (1997).

41. Orlandi, G., Zerbetto, F. \& Zgierski, M. Z. Theoretical analysis of spectra of short polyenes. Chem. Rev. 91, 867-891 (1991).

42. Orlandi, G. \& Zerbetto, F. Vibronic coupling in 1,2,4,5-tetrafluorobenzene: the double-minimum potential of the butterfly motion (Q11) in the state $\mathrm{S}_{1}$. J. Phys. Chem. 91, 4238-4240 (1987).

43. Hamm, P., Ohline, S. M. \& Zinth, W. Vibrational cooling after ultrafast photoisomerization of azobenzene measured by femtosecond infrared spectroscopy. J. Chem. Phys. 106, 519-529 (1997).
44. Dyck, R. H. \& McClure, D. S. Ultraviolet spectra of stilbene, p-monohalogen stilbenes, and azobenzene and the trans to cis photoisomerization process. J. Chem. Phys. 36, 2326-2345 (1962).

45. Andersson, J.-Å., Petterson, R. \& Tegnér, L. Flash photolysis experiments in the vapour phase at elevated temperatures I: spectra of azobenzene and the kinetics of its thermal cis-trans isomerization. J. Photochem. 20, 17-32 (1982).

46. Biancalana, A. et al. Direct observation of a $220 \mathrm{~cm}^{-1}$ structure in the lowest $\pi-\pi^{*}$ absorption band in the vapour phase of trans-azobenzene. Spectrochim. Acta A Mol. Biomol. Spectrosc. 55, $2883-2887$ (1999).

47. Fujino, T., Arzhantsev, S. Y. \& Tahara, T. Femtosecond time-resolved fluorescence study of photoisomerization of trans-azobenzene. J. Phys. Chem. A 105, 8123-8129 (2001).

48. Frisch, M. J. et al. Gaussian 09, Revision A.01 (Gaussian Inc., 2009).

49. Tsuji, T., Takashima, H., Takeuchi, H., Egawa, T. \& Konaka, S. Molecular structure and torsional potential of trans-azobenzene. A gas electron diffraction study. J. Phys. Chem. A 105, 9347-9353 (2001).

\section{Acknowledgements}

This work was supported by The Netherlands Organization for Scientific Research (NWO). S.A. would like to thank the Deutsche Akademie der Naturforscher Leopoldina-German National Academy of Sciences for a Leopoldina research fellowship (grant number LPDS 2011-18)

\section{Author contributions}

E.M.M.T. designed and performed the experiments, performed the calculations and analysed the data. S.A. assisted with the experiments, calculations and their interpretation. S.S. and A.V. designed and performed initial experiments on the $S_{2}\left(\pi \pi^{*}\right)$ state that formed the basis for the experiments reported here. F.Z. helped with the interpretation of the experimental data. W.J.B. designed the experiments and supervised the experiments, calculations and data analysis. E.M.M.T. and W.J.B. co-wrote the manuscript with input from S.A. and F.Z

\section{Additional information}

Supplementary Information accompanies this paper at http://www.nature.com/ naturecommunications

Competing financial interests: The authors declare no competing financial interests

Reprints and permission information is available online at http://npg.nature.com/ reprintsandpermissions/

How to cite this article: Tan, E. M. M. et al. Fast photodynamics of azobenzene probed by scanning excited-state potential energy surfaces using slow spectroscopy. Nat. Commun. 6:5860 doi: 10.1038/ncomms6860 (2015).

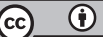

This work is licensed under a Creative Commons Attribution 4.0 International License. The images or other third party material in this article are included in the article's Creative Commons license, unless indicated otherwise in the credit line; if the material is not included under the Creative Commons license, users will need to obtain permission from the license holder to reproduce the material. To view a copy of this license, visit http://creativecommons.org/licenses/by/4.0/ 